\title{
What New Lens Technologies Are on the Horizon?
}

\section{This editorial is based on the "SYM-1: Advanced Cataract Surgery: Achieving 20/Happy in 2020" session at the American Society of Cataract and Refractive Surgery Virtual Annual Meeting}

Aman Mittal and Sumit Garg

Gavin Herbert Eye Institute, University of California, Irvine, Irvine, CA, USA

DOI: https://doi.org/10.17925/OPHT.2021.15.1.6

C urrent presbyopia-correcting intraocular lens (IOL) technologies have evolved since their introduction in the mid-2000's. Since then, there has been a steady increase in the number of new lenses that surgeons have at their disposal to help their patients. Depending on the lens design, these lenses do not always provide excellent and reproducible uncorrected near, intermediate, and distance vision in all patients. Newer innovations in presbyopia-correcting lenses include: extended depth of focus, trifocal, and innovative combination optics lenses. Additionally, on the horizon are modular IOLS, which are comprised of separate parts that can move independently to correct presbyopia or to facilitate IOL exchange. Each of these technologies has the potential to improve patients' visual function following cataract surgery in new and exciting ways.

\section{Keywords}

Presbyopia-correcting intraocular Iens (IOL), Modular IOL, extended depth of focus IOL, multifocal IOL

Disclosure: Aman Mittal has no financial or nonfinancial relationships or activities to declare in relation to this article. Sumit Garg is a consultant to LensGen and Johnson \& Johnson Vision.

Review Process: Double-blind peer review.

Compliance with Ethics: This study involves a review of the literature and did not involve any studies with human or animal subjects performed by any of the authors.

Authorship: The named authors meet the International Committee of Medical Journal Editors (ICMJE) criteria for authorship of this manuscript, take responsibility for the integrity of the work as a whole, and have given final approval for the version to be published. Access: This article is freely accessible at touchOPHTHALMOLOGY.com (c) Touch Medical Media 2020 Received: 14 September 2020

Accepted: 24 November 2020

Published Online: 25 May 2021

Citation: touchREVIEWS in Ophthalmology. 2021; 15(1):6-8

Corresponding Author: Sumit (Sam) Garg Gavin Herbert Eye Institute, University of California, Irvine, 850 Health Sciences Road, Irvine, CA 92697-4375, USA. E: gargs@uci.edu

Support: No funding was receiving in the publication of this article.
Cataract surgery has undergone a significant transformation in the last 70 years, becoming a safer and more reliable treatment with better visual outcomes. An important part of this transformation has been the advent of new intraocular lens (IOL) technologies, starting with the first IOL made of polymethyl methacrylate and implanted by Sir Harold Ridley in 1949. ${ }^{1}$ Modern-day cataract surgery in the USA most commonly consists of phacoemulsification for cataract removal and implantation of a foldable hydrophobic acrylic IOL. Advances in lens technology have included toric lenses for astigmatism correction, and multifocal, trifocal and extended depth of field lenses for presbyopia correction. No lens, however, has all the characteristics of the ideal IOL - providing excellent, predictable and reproducible uncorrected near, intermediate and distance vision with minimal modifications to current standard cataract surgery and without significant complications. The goal of cataract surgeons is to provide patients with spectacle-free vision, free of distortions and dysphotopsias, which current IOL technology cannot always provide.

\section{Extended depth of focus intraocular lenses}

Extended depth of focus (EDOF) IOLs are presbyopia-correcting lenses that aim to enhance intermediate and near vision. EDOF IOLS achieve this by creating single, elongated focus as opposed to overlapping near and far images, a method employed by multifocal IOLs. The Tecnis Symfony (Johnson \& Johnson Vision, Santa Ana, CA, USA) is a diffractive EDOF lens, but due to the diffractive optics has increased dysphotopsias and reduced contrast sensitivity compared with a monofocal lens. ${ }^{2}$ New monofocal lenses that provide additional intermediate vision are designed with a progressive power change and higher order aspheric surface that adds power towards the center of the lens. This design minimizes dysphotopsias and does not split light like previous EDOF and multifocal lenses while still partially extending the depth of vision. ${ }^{3}$ The Tecnis Eyhance (Johnson \& Johnson Vision, Santa Ana, CA, USA), is one such monofocal lens, which is now available in Europe and the USA. Initial studies show this lens provides comparable distance vision and rate of dysphotopsia with enhanced intermediate vision compared with standard monofocal lenses. ${ }^{4}$

Approved by the FDA in February 2020, the AcrySof IQ Vivity (Alcon, Fort Worth, TX, USA) is an EDOF lens that is based on a monofocal platform, but has a center elevation on the anterior surface with a different power to allow extended depth of focus and without diffractive elements. Because the lens does not split light but instead stretches and shifts the wavefront, it can be used in patients that would not be candidates for prior diffractive EDOF/multifocal lenses. ${ }^{5}$ This lens is available in Europe and the USA. 
EDOF can also be achieved by combining small aperture optics with a monofocal lens to treat patients with cataracts. ${ }^{6}$ The IC-8 (AcuFocus, Irvine, CA, USA) uses a small aperture and has been successful in providing EDOF and allowing spectacle independence in over $50 \%$ of patients implanted with the IOL in a recent study. ${ }^{6}$ The pinhole optics also provide a benefit in corneas with irregular astigmatism and high levels of aberrations, such as those with previous history of radial keratotomy or laser vision correction. Available outside the USA, IC-8 recently completed its FDA clinical trial and has submitted a premarket approval application to the FDA.?

\section{Combination extended depth of focus intraocular lenses and multifocal technology}

The Tecnis Synergy lens (Johnson \& Johnson Vision, Santa Ana, CA, USA) combines the diffractive EDOF technology of the Tecnis Symfony with multifocality to create a continuous range of vision from distance to $33 \mathrm{~cm}$, without the drop-out at intermediate distances seen with many previous multifocal and trifocal designs. Whereas the Symfony lens uses diffractive optics to achieve EDOF from distance to about 60-65 cm, the Synergy lens uses a combination of diffractive EDOF and multifocal optics to provide a near focal point of $33 \mathrm{~cm}$, a focal point at distance and an EDOF range from about 33 to $80 \mathrm{~cm}$ for intermediate vision. ${ }^{~}$ The lens is available in Europe, Australia and New Zealand.

\section{Trifocal lenses}

Trifocal lenses are multifocal lenses that use diffractive optics to create three distinct focal points, providing near, intermediate and distance vision. The PanOptix lens (Alcon, Fort Worth, TX, USA) is a trifocal lens that was launched in the USA in late 2019, and is the only FDA-approved trifocal lens available in the USA. The EnVista trifocal (Bausch + Lomb, Rochester, NY, USA), available in Europe since 2017, is in FDA clinical trials for registration in the USA (NCT NCT03603600).

\section{Modular intraocular lenses}

A new generation of IOLs is in the pipeline that include multiple separate components, called modular IOLs. Theodore Werblin initially proposed the concept of modular IOLs in the 1990s, and since then, multiple different lenses have been developed and are in various stages of production. Modular IOLs have a different set of trade-offs than traditional singleand three-piece IOLs, and can allow for presbyopia correction, easier IOL exchange, decreased rate of posterior capsular opacification and even implantation of other intraocular technologies, such as sensors. Another advantage is the reduced need for components to be kept in stock in the operating room; separate components can be used to correct sphere, cylinder and presbyopia, reducing the total inventory needed. ${ }^{10}$ Facilitating IOL exchange is important and can have significant effects on treatment of post-cataract surgery refractive error correction.

ClarVista Medical has developed a modular IOL called the Harmoni. This lens is again a foldable acrylic dual IOL with hydrophobic base and optic components. ${ }^{11}$ The base in this case has no optic and serves as a scaffold for the optic. The base is $13.0 \mathrm{~mm}$ in diameter including the haptics, and the central body of the base has a diameter of $8.5 \mathrm{~mm}$. The body is open in the centre, and the $5.8 \mathrm{~mm}$ diameter optic is placed within the body to form the final lens. Both parts of this IOL are designed to be placed within the capsular bag. The base has a square edge on the anterior and posterior sides, designed to reduce the rate of capsular opacification. The rate is further reduced by the large size of the base, preventing central migration of proliferative lens epithelial cells. The IOL is designed to allow for easy IOL exchange or upgrade if needed.
The Gemini Refractive Capsule ${ }^{\mathrm{TM}}$ (Omega Ophthalmics ${ }^{\oplus}$, Lexington, $\mathrm{KY}, \mathrm{USA}$ ) is a single-piece implant that acts somewhat similarly to the base of the Harmoni, but with the unique capability of accepting any modern lens design, making it part of a modular system. The implant is placed within the capsular bag, propping it open, and is open centrally. Any ordinary IOLs can be placed within the central opening created. By opening the capsular bag, the rate of posterior capsule opacification formation is reduced, and IOL exchange is facilitated as the Gemini maintains space and prevents adhesion of the lens to the capsular bag. Omega Ophthalmics is developing a hydrophobic acrylic optic that can be used with the Gemini alone or with a traditional IOL to refine refractive outcomes. ${ }^{12}$

FluidVision $^{\circledast}$ (PowerVision, Alcon, Geneva, Switzerland) is an accommodating IOL design consisting of a clear deformable optic filled with fluid attached to peripheral reservoirs that change the shape and refractive power of the lens in response to accommodative effort. This design is currently under development. ${ }^{13}$

Atia Vision (Shifamed, Campbell, CA, USA) is developing a modular IOL that acts to correct presbyopia. It consists of a back and front lens similar to other designs. The back lens sits within the capsular bag, filling it and maintaining contact with the posterior surface of the bag. It changes shape with the contraction and relaxation of the ciliary body, thereby increasing and decreasing its power. The front lens is a fixed power optic that is selected based on the patient's biometry to minimize residual refractive error. This design allows for natural accommodation of the IOL and selection of the appropriate power for each patient. The Atia Vision lens is currently undergoing in-human trials in Europe. ${ }^{14}$

The Opira lens (ForSight Labs, Menlo Park, CA, USA) is a sulcus-based accommodating IOL that fixes haptics in the capsulorhexis, putting them in direct contact with the ciliary body. Contractions pump fluid into the anterior optic, changing its shape and power..$^{15}$

Another new modular IOL that provides presbyopia correction by allowing accommodation is the Juvene ${ }^{\text {TM }}$ Iens (LensGen, Irvine, CA, USA). The Juvene IOL consists of a base component with a fixed power optic that fills the capsular bag, and a fluid-filled anterior lens that changes power with contraction and relaxation of the ciliary body. A unique advantage of the Juvene is that the lens fills the entire capsular bag, anterior to posterior. This allows more accurate prediction of effective lens position, increases rotational stability, prevents capsular opacification, and can potentially reduce vitreous movement, reducing the rate of posterior vitreous detachment and retinal tears or detachments. The anterior lens power sits within the base component and is held in place by three tabs. The power of the anterior lens can be selected based on preoperative biometry, and a toric version can be implants in patients with corneal astigmatism. The platform also allows for future developments such as implants that can be used to deliver intraocular medications and implantable sensors. Implanting the Juvene IOL is done in the same fashion as standard monofocal one-piece IOLs, via a clear corneal incision of $3.0 \mathrm{~mm}$. The anterior lens is inserted after the base lens and is set within the base using a second instrument. ${ }^{16}$ The Juvene has been implanted in patients, including those in the ongoing GRAIL study. In this study, the Juvene IOL was implanted in 44 eyes and patients were followed for 12 months postoperatively. Preliminary data have been reported, demonstrating the benefits of the Juvene IOL. Ten eyes were examined for rotational stability at 3 months postoperatively, and the mean rotation was found to be $1.7^{\circ} \pm 0.9^{\circ}$. Of 20 eyes with Juvene 
IOLs, the monocular defocus curves showed mean UDVA of 20/40 or greater from $-2.5 \mathrm{D}$ to $+1.0 \mathrm{D}$, and 15 patients underwent binocular Juvene implantation (30 eyes). Of these 15 patients, none reported visual complaints on the McAlinden quality of vision questionnaire. After 6 months, the binocular defocus curves showed mean UDVA of 20/40 or greater from -3.0 D to +1.5 D, demonstrating the Juvene IOL's presbyopia- correcting capability. ${ }^{17}$ The final results of the GRAIL study are expected to be published in the near future.

Our options for presbyopia correction continue to expand. There are many near-term and long-term options that promise to improve on current technologies for our patients. The future certainly looks exciting! $\square$
1. Apple DJ, Sims J. Harold Ridley and the invention of the intraocular lens. Surv Ophthalmol. 1996;40:279-92.

2. Liu J, Yi D, Yan W. Efficacy and safety of extended depth of focus intraocular lenses in cataract surgery: a systematic review and meta-analysis. BMC. 2019;19.1:1-10

3. Mencucci R, Cennamo M, Venturi D, et al. Visual outcome, optical quality, and patient satisfaction with a new monofocal $1 \mathrm{OL}$, enhanced for intermediate vision: preliminary results. 1 Cataract Refract Surg. 2020;46.3:378-87. 4. Auffarth GU, Gerl M, Tsai L, et al. Clinical evaluation of a new (in patients with cataract. J Cataract Refract Surg. 2021;47.2:184-91.

Schallhorn, JM. Multifocal and extended depth of focus intraocular lenses: A comparison of data from the United State Food and Drug Administration Premarket Approval Trials. $J$ Refract Surg. 2021;37.2: 98-104.

6. Hooshmand J, Allen P, Huynh T, et al. Small aperture IC-8 intraocular lens in cataract patients: achieving extended depth of focus through small aperture optics. Eye (Lond). 2019;33:1096-103.

7. AcuFocus. AcuFocus Announces Submission of its US
Premarket Approval Application to the FDA for its IC-8® Smal Aperture IOL. Feb 2021. Available at: www.acufocus.com us/sites/default/files/MKU\%20651\%20Rev\%20A\%20-\%20 AcuFocus\%20PMA\%20Press\%20Release\%20Final\%20for\%20 Release.pdf (accessed 10 April 2021).

8. Barsam A, Carones F. Novel IOL Technologies Heighten the Patient Experience. Jan 2020. Available at: https:// crstodayeurope.com/articles/2020-jan/novel-iol-technologiesheighten-the patientexperiencel (accessed 10 April 2021).

9. Werblin TP Multicomponent intraocular lens J Refract Surg. 1996;12:187-9.

10. Portaliou DM, Grentzelos MA, Pallikaris IG. Multicomponent intraocular lens implantation: two-year follow-up. J Catarac Refract Surg. 2013:39:578-84.

11. Werner, L. The Harmoni Modular IOL System. Cataract \& Refractive Surgery Today May 2018. Available at: https:// crstodayeurope.com/articles/2018-may/the-harmoni-modulariol-system/ (accessed August 24, 2020)

12. Wörtz G. Overcoming the butterfly effect in refractive cataract surgery. 2018. Available at: https://crstoday.com/articles/2018may/overcoming-the-butterfly-effect-in-refractive-cataract- surgery/ (accessed 31 March 2021)

13. Scholl J. FluidVision Accommodating IOL. Presented at Wavefront \& Presbyopic Refractive Corrections Congress, February 2008

14. Kent C. Accommodating IOLs: two more possibilities. 2019 Available at: www.reviewofophthalmology.com/article/ accommodating-iols-two-more-possibilities (accessed 31 March 2021).

15. Naseri, A. ForSight Vision6: Accommodating IOL. Presented at OIS@ASCRS 2019; May 2019. Available at: https://ois.net/ wp-content/uploads/2019/05/Presbyopia_ForSightV6.pdf (accessed 2 October 2020)

16. Lenier S. Innovation case study: LensGen focusing on a complete presbyopia correcting IOL for declining vision. Available at: https://ois.net/innovation-case-study-lensgenfocusing-on-a-complete-presbyopia-correcting-iol-fordeclining-vision/ (accessed 31 March 2021).

17. Garg S. What new lens technologies are on the horizon? presented at the ASCRS Virtual Annual Meeting, May 2020 